\title{
Analysis of phenotype, enzyme activity and genotype of Chinese patients with POMT1 mutation
}

\author{
Haipo Yang ${ }^{1,6}$, Hiroshi Manya ${ }^{2,6}$, Kazuhiro Kobayashi ${ }^{3}$, Hui Jiao ${ }^{1}$, Xiaona $\mathrm{Fu}^{1}$, Jiangxi Xiao ${ }^{4}$, Xiaoqing $\mathrm{Li}^{5}$, \\ Jingmin Wang ${ }^{1}$, Yuwu Jiang ${ }^{1}$, Tatsushi Toda ${ }^{3}$, Tamao Endo ${ }^{2}$, Xiru $\mathrm{Wu}^{1}$ and Hui Xiong ${ }^{1}$
}

Protein 0-mannosyltransferase 1 (POMT1) is a glycosyltransferase involved in $\alpha$-dystroglycan glycosylation. POMT1 mutations cause a wide spectrum of clinical conditions from Walker-Warburg syndrome (WWS), which involves muscle, eye and brain abnormalities, to mild forms of limb-girdle muscular dystrophy with mental retardation. We aimed to elucidate the impact of different POMT1 mutations on the clinical phenotype. We report five Chinese patients with POMT1 mutations: one had a typical clinical manifestation of WWS, and the other four were diagnosed with congenital muscular dystrophy with mental retardation of varying severity. We analyzed the influence of the POMT1 mutations on POMT activity by assaying the patients' muscles and cultured skin fibroblasts. We demonstrated different levels of decreased POMT activity that correlated highly with decreased $\alpha$-dystroglycan glycosylation. Our results suggest that POMT activity is inversely proportional to clinical severity, and demonstrate that skin fibroblasts can be used for differential diagnosis of patients with $\alpha$-dystroglycanopathies. We have provided clinical, histological, enzymatic and genetic evidence of POMT1 involvement in five unrelated Chinese patients.

Journal of Human Genetics (2016) 61, 753-759; doi:10.1038/jhg.2016.42; published online 19 May 2016

\section{INTRODUCTION}

Protein O-mannosyltransferases 1 and 2 (POMT1 and POMT2), two closely related proteins, are type III transmembrane (TM) glycosyltransferases that colocalize in the endoplasmic reticulum. Taken together, they catalyze the O-linked addition of a mannose from a dolichol-linked precursor onto a serine or threonine residue of a polypeptide. ${ }^{1-3}$ POMT1 mutations have been reported to lead to Walker-Warburg syndrome (WWS), congenital muscular dystrophy (CMD) with mental retardation and limb-girdle muscular dystrophy $2 \mathrm{~K}$ (LGMD2K). ${ }^{4-7}$ WWS is a cerebro-oculo muscular syndrome whose clinical manifestations include CMD and brain abnormalities such as type II (cobblestone) lissencephaly, hydrocephalus and cerebellar malformation. ${ }^{8}$ Ocular abnormalities include microcornea, shallow anterior chamber, lens defects, retinal detachment, glaucoma and buphthalmos. Compared with WWS, patients who have CMD with mental retardation usually have delayed mental and motor development, and normal or abnormal brain magnetic resonance imaging (MRI); however, there is usually no ocular abnormality. LGMD2K is characterized by late-onset and mild clinical symptoms, but patients usually have microcephalus and mental retardation. ${ }^{7,9}$ In this study, we report the results of POMT1 mutation analysis in five patients and analyze the correlation between POMT activity in dermal fibroblasts and the clinical severity of patients with the different POMT1 mutations.

\section{PATIENTS AND METHODS}

Patients and clinical data

The inclusion criteria were clinical diagnosis of muscular dystrophy characterized by muscle weakness or hypotonia with early-onset, delayed developmental milestones and mental retardation. Clinical and laboratory data, including ophthalmological findings, motor and language development, serum creatine kinase (CK) levels, brain MRI and family history were reviewed. Open muscle biopsy was performed in some patients to ensure that they have $\alpha$-dystroglycan $(\alpha-D G)$ myopathy. The study was approved by the Ethics Committee of the Peking University First Hospital. Informed written consent was obtained for participation in the study from the parents or guardians of the children, as well as for the publication of control medical data.

Gene mutation analysis

Based on the clinical features and elevated serum CK levels, next-generation sequencing (NGS) and Sanger sequencing were used to test for gene mutation. Genomic DNA was extracted from peripheral blood lymphocytes using standard protocols. For NGS, the genomic DNA was first converted into a DNA library and then amplified. Myopathy-related genes, including 169 genes in the panel, were selected for using the SureSelect Target Enrichment Kit (Agilent Technologies, Santa Clara, CA, USA). The sequencing was carried out on a GAIIx from Illumina (San Diego, CA, USA). The results were transferred to recognizable base sequence using CASAVA1.8.24 software (Illumina). Clean paired-end reads were aligned on the human reference genome build hg19. ANNOVAR was used to annotate sequences and to call insertion-deletions and single-nucleotide polymorphisms. In silico prediction of

\footnotetext{
${ }^{1}$ Department of Pediatrics, Peking University First Hospital, Beijing, China; ${ }^{2}$ Molecular Glycobiology, Research Team for Mechanism of Aging, Tokyo Metropolitan Geriatric Hospital and Institute of Gerontology, Tokyo, Japan; ${ }^{3}$ Division of Neurology/Molecular Brain Science, Kobe University Graduate School of Medicine, Kobe, Japan; ${ }^{4}$ Department of Radiology, Peking University First Hospital, Beijing, China and ${ }^{5}$ Department of Child Ophthalmology, Peking University First Hospital, Beijing, China

${ }^{6}$ These authors contributed equally to this work.

Correspondence: Professor H Xiong, Department of Pediatrics, Peking University First Hospital, No. 1 Xi'an Men Street, West District, Beijing 100034, China.

E-mail: xh_bjbj@163.com

Received 9 August 2015; revised 4 March 2016; accepted 30 March 2016; published online 19 May 2016
} 
pathogenicity for novel missense variants was performed using Polyphen2.2.2 (http://genetics.bwh.harvard.edu/pph2/) and SIFT (http://sift.jcvi.org/). Singlenucleotide polymorphisms deposited in the 1000 Genomes Database or in dbSNP (build 132) were removed. Phylogenetic conservation analysis of missense mutations was also performed using several organisms.

\section{Muscle pathology}

Open biopsy of the gastrocnemius muscle was performed, and samples were fixed in isopentane cooled in liquid nitrogen. Routine histochemical staining was performed. Frozen sections $(6-8 \mu \mathrm{m})$ from the muscle biopsy specimens were fixed with $4 \%$ paraformaldehyde at room temperature for $10 \mathrm{~min}$. Nonspecific binding was reduced by a 30-min incubation with $10 \%$ goat serum (Jackson ImmunoResearch, West Grove, PA, USA) in phosphate-buffered saline (PBS). Sections were then incubated at $4{ }^{\circ} \mathrm{C}$ overnight with a primary $\alpha$-DG antibody, IIH6 (1:200; Chemicon, Temecula, CA, USA), washed three times in PBS and then incubated with a rabbit anti-mouse fluorescent antibody (1:200; Jackson ImmunoResearch) at room temperature for $1 \mathrm{~h}$. Sections were observed using an Olympus Fluoview ver 1.6b viewer (Olympus, Tokyo, Japan).

\section{POMT enzyme assay}

Cell culture and preparation of microsomal membrane fraction. Fibroblasts were maintained in Dulbecco's modified Eagle's medium (Invitrogen, Carlsbad, CA, USA) supplemented with $20 \%$ fetal bovine serum, $2 \mathrm{~mm}$ L-glutamine, $100 \mathrm{U} \mathrm{ml}^{-1}$ penicillin, $50 \mathrm{mg} \mathrm{ml}^{-1}$ streptomycin and $0.5 \mathrm{U} \mathrm{ml}^{-1}$ amphotericin $\mathrm{B}$ at $37^{\circ} \mathrm{C}$ with $5 \% \mathrm{CO}_{2}$. Fibroblasts and muscle samples were homogenized in $10 \mathrm{~mm}$ Tris- $\mathrm{HCl}$ ( $\mathrm{pH} 7.4$ ), $1 \mathrm{~mm}$ EDTA, $250 \mathrm{~mm}$ sucrose, $1 \mathrm{~mm}$ dithiothreitol and protease inhibitor cocktail $\left(3 \mu \mathrm{g} \mathrm{ml}^{-1}\right.$ pepstatin $\mathrm{A}, 1 \mu \mathrm{g} \mathrm{ml}{ }^{-1}$ leupeptin, $1 \mathrm{~mm}$ benzamidine-HCl, $1 \mathrm{~mm}$ phenylmethylsulfonyl fluoride). Normal control fetal muscle was obtained from a fetus that had been aborted for reasons other than a neuromuscular disorder, and written informed consent was obtained for this use. After centrifugation for $10 \mathrm{~min}$ at $900 \mathrm{~g}$, the supernatant was centrifuged at $100000 \mathrm{~g}$ for $1 \mathrm{~h}$. The pellet was used as the microsomal membrane fraction. Protein concentrations were determined using a bicinchoninic acid assay (Pierce, Rockford, IL, USA).

POMT activity assay. As described previously, ${ }^{10}$ POMT activity is based on the amount of $\left[{ }^{3} \mathrm{H}\right]$ mannose transferred to a glutathione $S$-transferase fusion $\alpha$-DG. Briefly, the reaction mixture contained $20 \mathrm{~mm}$ Tris- $\mathrm{HCl}(\mathrm{pH} 8.0$ ), 200 nм [ $\left.{ }^{3} \mathrm{H}\right]$ mannosyl phosphoryl dolichol (1 25000 d.p.m. pmol ${ }^{-1}$; American Radiolabeled Chemicals, St Louis, MO, USA), 2 mм 2-mercaptoethanol, $10 \mathrm{~mm}$ EDTA, $0.5 \% n$-octyl-D-thioglucoside, $10 \mu \mathrm{g}$ glutathione $S$-transferase fusion $\alpha$-DG and enzyme source (60 $\mu \mathrm{g}$ (fibroblasts) or $80 \mu \mathrm{g}$ (muscle) microsomal membrane fraction) in a total volume of $20 \mu \mathrm{l}$. After incubation at $22{ }^{\circ} \mathrm{C}$ (fibroblasts, $1 \mathrm{~h}$; muscle, $3 \mathrm{~h}$ ), the reaction was stopped by adding $150 \mu \mathrm{l}$ PBS containing $1 \%$ Triton X-100 and the mixture was centrifuged at $10000 \mathrm{~g}$ for $10 \mathrm{~min}$. The supernatant was removed, mixed with $400 \mu \mathrm{l}$ PBS containing $1 \%$ Triton X-100 and $10 \mu \mathrm{l}$ glutathione sepharose 4B beads (GE Healthcare Bio-Sciences, Piscataway Township, NJ, USA), rotated at $4{ }^{\circ} \mathrm{C}$ for $1 \mathrm{~h}$ and washed three times with $20 \mathrm{~mm}$ Tris- $\mathrm{HCl}(\mathrm{pH} 7.4)$ containing $0.5 \%$ Triton $\mathrm{X}-100$. The radioactivity adsorbed to the beads was measured using a liquid scintillation counter.

$N$-acetylglucosaminyltransferase I (GnT-I) activity was detected using a $20-\mu \mathrm{l}$ reaction mixture containing $100 \mathrm{~mm} 2$-( $N$-morpholino) ethanesulfonic acid buffer, $10 \mu \mathrm{M}$ pyridylaminated $\mathrm{Man}_{5} \mathrm{GlcNAc}_{2}$ (Takara Bio, Otsu, Japan), 2 mм uridine diphosphate- $\mathrm{N}$-acetylglucosamine, $5 \mathrm{~mm}$ adenosine monophosphate, $0.5 \%$ Triton X-100, $0.2 \%$ bovine serum albumin, $20 \mathrm{~mm} \mathrm{MnCl}_{2}$ and enzyme source $(60 \mu \mathrm{g}$ (fibroblasts) or $80 \mu \mathrm{g}$ (muscle) microsomal membrane fraction) at $37^{\circ} \mathrm{C}$ for $3 \mathrm{~h}$. The samples were analyzed by reverse-phase high-performance liquid chromatography using a Cosmosil 5C18-AR-II column $\left(4.6 \times 250 \mathrm{~mm}^{2}\right.$; Nacalai Tesque, Kyoto, Japan). The solvent was $100 \mathrm{~mm}$ ammonium acetate buffer ( $\mathrm{pH}$ 6.0) containing $0.15 \%$ 1-butanol, and the substrate and product were isocratically separated. Fluorescence was detected using a RF-10AXL detector (Shimadzu Corp., Kyoto, Japan) at excitation and emission wavelengths of 320 and $400 \mathrm{~nm}$, respectively.

\section{Prenatal diagnosis}

Prenatal diagnosis was performed on two further pregnancies of the mother of Patient 5 (subsequent to that of Patient 5) at 13 and 23 gestational weeks, respectively. A $20-\mathrm{ml}$ amniotic fluid sample was collected by amniocentesis under ultrasound guidance at 23 weeks and divided into a $12-\mathrm{ml}$ sample for extracting DNA from free fetal cells (amniocytes), and an 8-ml sample for culture. Chorionic villus sampling was performed at 13 weeks of gestation and DNA was extracted. Before DNA sequencing, maternal cell contamination was excluded by PCR linkage analysis of polymorphic microsatellite markers, in which three DNA markers linked to the X chromosome (AR, DXS6797, DXS6807) were used. The sex of the fetus was determined by karyotype analysis and PCR amplification of the sex-determining region Y (NM_003140). Fetal DNA was extracted using a Wizard Genomic DNA Purification Kit (Promega, Madison, WI, USA) according to the manufacturer's instructions. Results of fetal mutation analysis were confirmed by PCR using the DNA extracted from the samples prepared above.

\section{Western blot analysis}

Muscle tissue was homogenized in $10 \mathrm{~mm}$ Tris- $\mathrm{HCl}$ (pH 7.4), $1 \mathrm{~mm}$ EDTA, $250 \mathrm{~mm}$ sucrose, $1 \mathrm{~mm}$ dithiothreitol and protease inhibitor cocktail ( $3 \mathrm{mg} \mathrm{m}^{-1}$ pepstatin $\mathrm{A}, 1 \mathrm{mg} \mathrm{ml}^{-1}$ leupeptin, $1 \mathrm{~mm}$ benzamidine- $\mathrm{HCl}$, $1 \mathrm{~mm}$ phenylmethylsulfonyl fluoride). After centrifugation for $10 \mathrm{~min}$ at $900 \mathrm{~g}$, the supernatant was centrifuged for $1 \mathrm{~h}$ at $100000 \mathrm{~g}$ and the protein concentration was determined using a bicinchoninic acid assay (Thermo Fisher Scientific, Waltham, MA, USA). The microsomal fractions $(20 \mu \mathrm{g})$ were separated by sodium dodecyl sulfate-polyacrylamide gel electrophoresis $(7.5 \%$ polyacrylamide gel), and proteins were transferred to a polyvinylidene difluoride membrane. The membrane was blocked in PBS containing 5\% skimmed milk and $0.05 \%$ Tween-20, incubated with IIH6 antibody and treated with horseradish peroxidase-conjugated anti-mouse immunoglobulin G. Proteins that bound to the antibody were visualized using an ECL Kit (GE Healthcare Bio-Sciences Corp.).

\section{RESULTS}

\section{Clinical characteristics}

Twenty-four pediatric patients attending Peking University First Hospital between 2008 and 2014 were clinically and/or pathologically diagnosed with $\alpha$-dystroglycanopathies and in five patients, mutations in POMT1 were identified. All patients are of Han ethnicity. The clinical characteristics of these five patients are summarized in Table 1.

Patient 1. Patient 1 presented with typical clinical signs of WWS. He had delayed psychomotor development after birth, muscle hypotonia, early joint contractures, glaucoma and microcornea. His serum CK level was moderately elevated and brain MRI revealed structural malformations, including a cerebellar cyst, bilateral dilatation of the lateral ventricle and cerebellar and brainstem dysplasia (Figure 1). He died at the age of 10 months but the cause of death was not determined.

Patient 2. Patient 2 was a 4-year-old boy with a weak cry at birth and motor and cognitive delay during the infantile period. He was born full term to a healthy mother who had one previous pregnancy and birth (G1P1). He could control his head movements when he was 8 months old, and sit unsupported at 14 months of age. When he was 2 years old, he could walk a few steps with help and could say 'mother' and 'father'. His head circumference was $46.2 \mathrm{~cm}$ when he was 4 years old, he had calf muscle hypertrophy and no significant joint contracture, but had mild muscle weakness and tendon reflexes were absent. Brain MRI revealed type II agyria deformities (Figure 1).

Patient 3. Patient 3 was a 13-year-old boy with a G1P1 mother and was born at full term. He had hypotonia and delayed motor milestones in the infantile period. He could control his head 
movements at 12 months and sit unsupported at 18 months. He could walk when he was 2 years old, but was unstably and fell easily. He could walk well when he was 4 years old, but was found to have pes cavus when he was 7 years old, which worsened over time. Consequently, he underwent Achilles tendon stretch surgery when he was 12 years old to improve the symptoms. Currently, he is able to climb up stairs without difficulty. He could say simple words when he was 2.5 years old, but he had slurred speech and obvious mental retardation. His head circumference was $48 \mathrm{~cm}$ when he was 8 years old and there was muscle weakness and an absence of tendon reflexes. $\mathrm{He}$ also had atypical retinitis pigmentosa and optic atrophy (Figure 2). His serum CK level was $2174 \mathrm{Ul}^{-1}$. Electromyography revealed a myopathic pattern, electrocardiogram showed sinus tachycardia and ultracardiography revealed mild left ventricular enlargement but with normal ejection fraction. Muscle biopsy demonstrated advanced dystrophic changes with a few necrotic and regenerating fibers with marked interstitial fibrosis.

Patient 4. Patient 4 was a 3-year and 3-month-old boy with a G2P1 mother, and was born at full term. The mother's history included G1P0 (spontaneous abortion). His developmental milestones were characterized by gross delay. At birth, he had a weak cry and difficulty in feeding. He could control his head movements at 8-9 months and sit unsupported at 18 months. At 3 years old, he could move forward slowly when he sat on a wooden bench, but could not stand alone or walk. He could speak simple words when he was 3 years old, and he drooled frequently. His head circumference was $45 \mathrm{~cm}$ when he was 2 years old, and he had muscle weakness, an absence of tendon reflexes and bilateral calf muscle hypertrophy. His serum CK level was $5566 \mathrm{Ul}^{-1}$. Brain MRI was performed at 2 years of age and was normal. Video electroencephalography revealed numerous continuous positive-phase sharp waves in the left occipital area that were aggravated by eye blinking. Occasionally, blinking induced negative-phase sharp waves in the left occipital area; the meaning of these responses in this patient requires follow-up studies.

Patient 5. Patient 5 was a 7-year-old boy born via full-term cesarean section. He had a weak cry at birth and his developmental milestones were characterized by obvious gross delay. He could control his head when he was 3.5 years old and sit unsupported when he was 4.5 years old. He was unable to walk but could speak simple words such as 'father' and 'mother' and understand simple instructions, but could not speak long sentences at present. Physical examination determined that he had facial muscle weakness and moderate visual loss. His head circumference at the age of 7 years was $49 \mathrm{~cm}$, and he had high arched palate, bilateral knee joint contracture, muscle weakness and an absence of tendon reflexes. Brain MRI revealed bilateral dilation of the lateral and fourth ventricles, brainstem hypoplasia and a cerebellar cyst (Figure 1). His serum CK level was $6620 \mathrm{Ul}^{-1}$.

Prenatal diagnosis was performed on two further pregnancies of the mother of Patient 5. The fetus of the first pregnancy had the same mutation as Patient 5, so the parents made the decision to abort the pregnancy. We performed western blot analysis and a POMT enzyme assay on muscle tissue from the aborted fetus (the results are detailed below).

POMT1 mutation analysis

We performed direct sequencing of POMT1 exons and their boundaries. Patient 5 was diagnosed using NGS. All five patients had compound heterozygous POMT1 mutations, most of which were known mutations. Patient 1, whose clinical manifestation was 

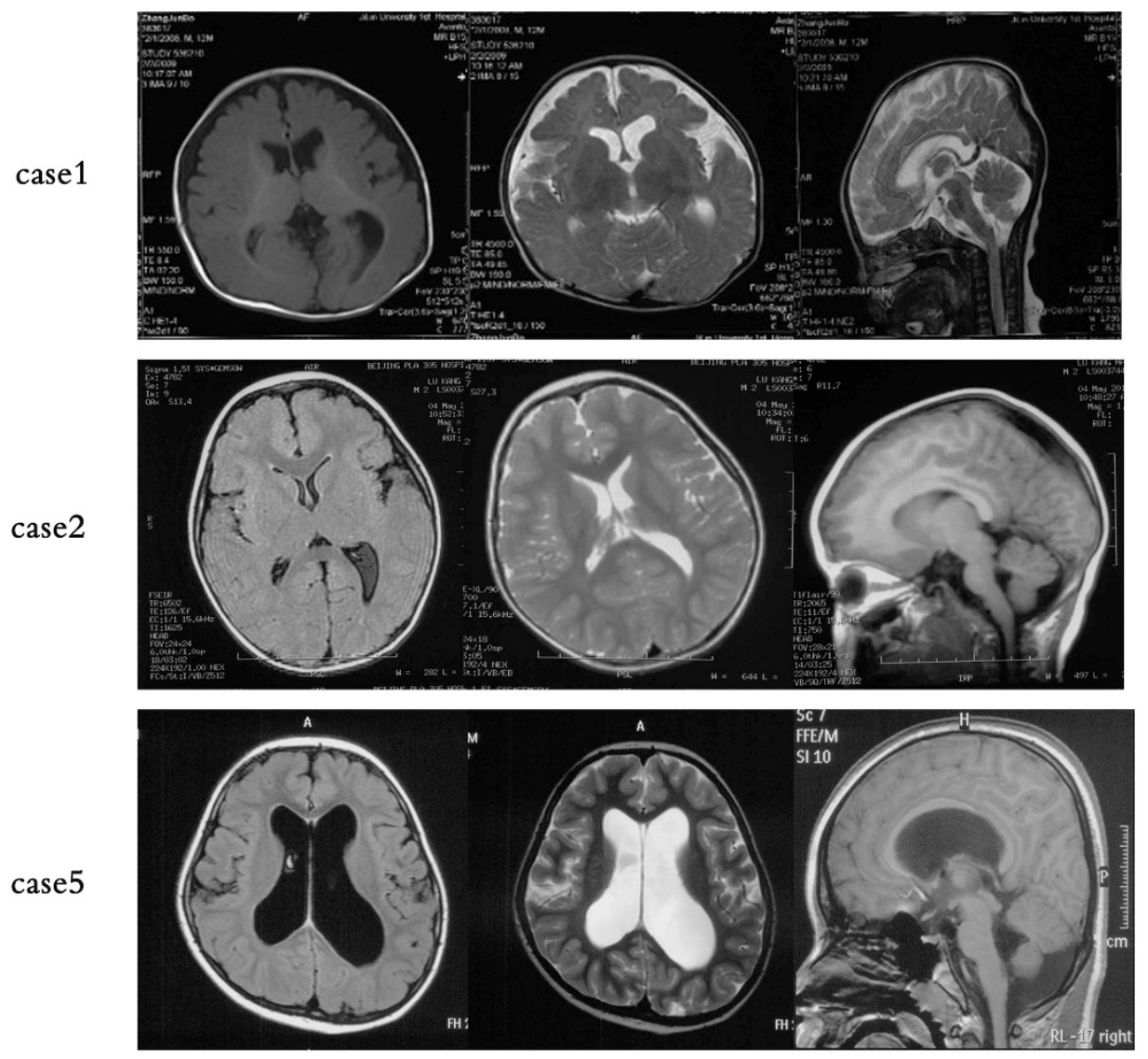

Figure 1 Brain magnetic resonance imaging (MRI). Brain MRI of Patients 1, 2 and 5 showed brainstem dysplasia, mild cerebellar dysplasia, cerebellar cysts, type II agyria deformities, bilateral dilation of the lateral and fourth ventricles and brain white matter hypomyelination changes.
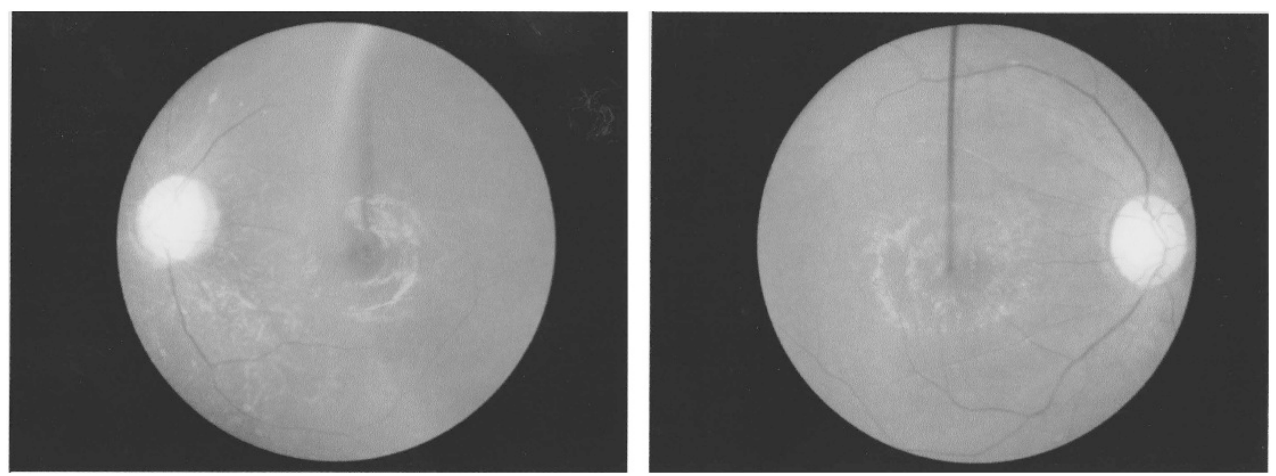

Figure 2 Ocular fundus examination. Ocular fundus examination of Patient 3, a 13-year-old boy, showed atypical retinitis pigmentosa and optic atrophy. A full color version of this figure is available at the Journal of Human Genetics journal online.

consistent with WWS, had a missense mutation in exon 5 (c.313C > T/p.Arg105Cys), and a frameshift mutation in exon 20 (c.2208delG/p.Trp736*). Patient 2 had a splice site mutation in exon 10 (c.1052+1G $>A$ ), and a missense mutation in exon 15 (c.1457G >C/p.Trp486Ser). Patient 3 had a mutation in exon 15 (c.1457G $>$ C/p.Trp486Ser) and a mutation in exon 3 (c.130G > A/p.Glu44Lys). Both Patients 2 and 3 had mild CMD with mental retardation because they could walk with or without help. Patient 4 had a small deletion in exon 3 (c.180-182del/p.Pro60del) and another missense mutation in exon 19 (c.1958C > A/p.Pro653Gln).
Patient 5 had a splice site mutation before the coding region of exon 2, c. $-32 \mathrm{~A}>\mathrm{G}$, and a frameshift mutation in exon 20, c.2207delG/p. Trp736*. Patients 4 and 5 had more severe CMD with mental retardation because they were unable to walk.

The genetic characteristics of these five patients are summarized in Table 1.

\section{Muscle pathology}

Muscle biopsies from patients 4 and 5, stained with hematoxylin-eosin, showed atrophic muscle fibers enclosed by markedly 


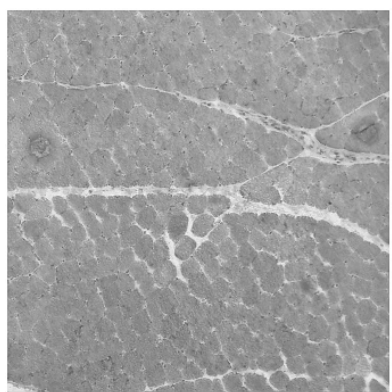

control

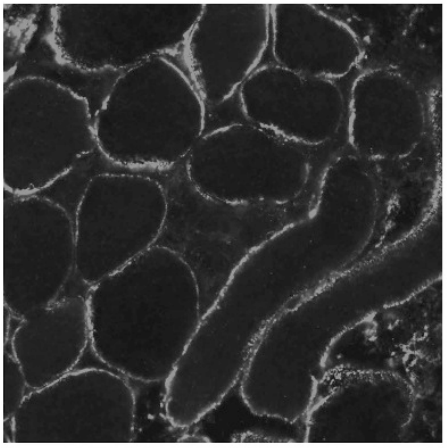

control

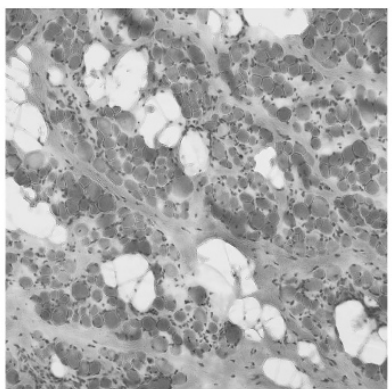

case 4

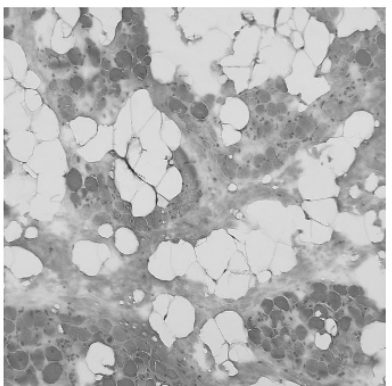

case 5

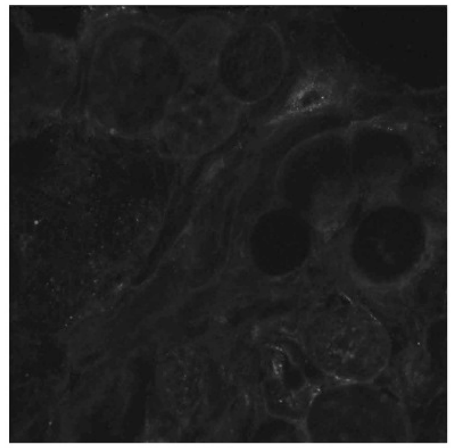

case 4

Figure 3 Hematoxylin and eosin (H\&E) staining. Skeletal muscle sections from Patients 4 and 5 and the normal control (top three photos). Dystrophic changes are evident in Patients 4 and 5: variability in the size of muscle fibers, fibrosis and fat replacement. Immunofluorescence staining of skeletal muscle tissue from the normal control and Patient 4 using an anti- $\alpha$-dystroglycan ( $\alpha$-DG) antibody (IIH6) (bottom two photos). An absence of $\alpha$-DG glycosylation staining was observed in Patient 4. A full color version of this figure is available at the Journal of Human Genetics journal online.

proliferated fibrous and fat tissue with no distinct fascicular structure, demonstrating advanced dystrophic changes (Figure 3). Immunofluorescence staining showed that glycosylated $\alpha$-DG was absent in the muscle cell membrane in Patient 4 (Figure 3).

\section{POMT activity assay}

POMT activity was measured in fibroblasts obtained from Patients 2-5 (Figure 4 and Table 2). A reduction in POMT activity was observed in all four patients. Interestingly, the remaining POMT activity differed according to clinical phenotype; Patients 2 and 3 had higher POMT activity than Patients 4 and 5. We also measured POMT activity in the muscle of the abortus and it was almost absent compared with the normal control. Comparable GnT-I activities were observed in control fetal muscle and in the abortus (Table 2), indicating that the absence of POMT activity in the abortus was not because of poor sample preparation. GnT-I is a Golgi glycosyltransferase and catalyzes the formation of GlcNAc $\beta 1-2 \mathrm{Man}$ in $N$-glycan biosynthesis. Because GnT-I is not involved in O-mannosylglycan biosynthesis, GnT-I activity was used as a control to normalize samples.

\section{Prenatal diagnosis}

All three X-chromosome markers (AR, DXS6797 and DXS6807) were informative in the family of Patient 5, with linkage analysis showing no maternal cell contamination in the amniocytes. In the first pregnancy, a female fetus carrying the same two POMT1 mutations as Patient 5 was detected and the fetus was aborted at 23 weeks of gestation. In the second pregnancy, a heterozygous splicing mutation, c. $-32 \mathrm{~A}>\mathrm{G}$, was detected in the fetus. This pregnancy continued to term and a healthy male baby was born.

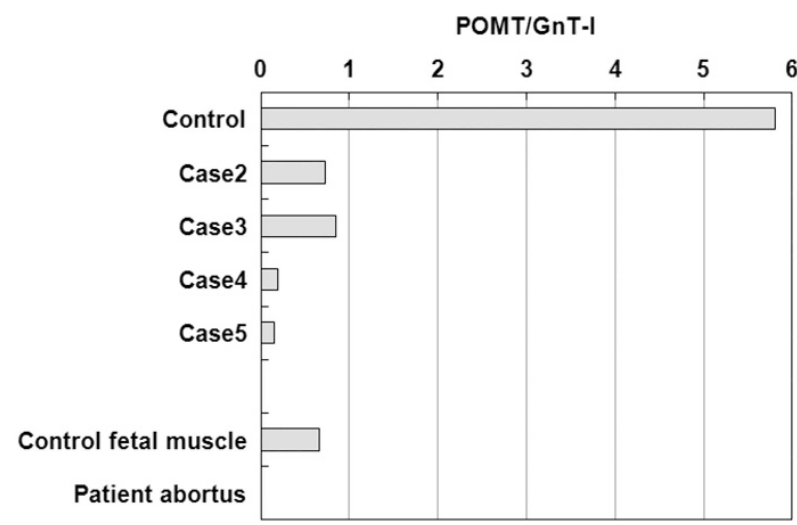

Figure 4 Ratios of protein 0-mannosyltransferase (POMT) activity to $\mathrm{N}$-acetylglucosaminyltransferase I (GnT-I) activity in fibroblasts from Patients $2-5$, and in abortus muscle. Compared with the normal control, POMT activity in the patients was decreased by varying degrees. POMT activity in the abortus muscle was absent compared with the normal control.

Western blot analysis of the abortus

Western blot analysis showed decreased $\alpha$-DG glycosylation in the abortus muscle compared with the control (Figure 5).

\section{DISCUSSION}

Mutations in POMT1 lead to WWS, CMD with mental retardation and LGMD2K. Here, we report five patients with a variety of mutations in POMT1. These patients had different clinical phenotypes, which included severe WWS and CMD with mental retardation. 
Table 2 POMT activity and GnT-I activity in fibroblasts and muscle

\begin{tabular}{|c|c|c|c|c|c|}
\hline Sample & POMT activity (pmol $h^{-1}$ per $\mathrm{mg}$ ) & $P O M T \pm$ s.d. & GnT-I activity ( $\mathrm{nmol} \mathrm{h}^{-1}$ per $\left.\mathrm{mg}\right)$ & GnT-I \pm s.d. & POMT/GnT-I \\
\hline Control & 0.54 & 0.083 & 0.09 & 0.010 & 5.80 \\
\hline Case 2 & 0.06 & 0.004 & 0.08 & 0.002 & 0.72 \\
\hline Case 4 & 0.02 & 0.004 & 0.12 & 0.010 & 0.19 \\
\hline Case 5 & 0.04 & 0.009 & 0.23 & 0.011 & 0.16 \\
\hline Control fetal muscle & 0.04 & 0.005 & 0.06 & 0.004 & 0.66 \\
\hline
\end{tabular}

Abbreviations: GnT-I, N-acetylglucosaminyltransferase I; POMT1, protein O-mannosyltransferase 1.

Average values of three (fibroblast) or two (muscle) independent experiments are shown. As GnT-I is not involved in 0 -mannosylglycan biosynthesis, GnT-I activity was used as a control to normalize samples.

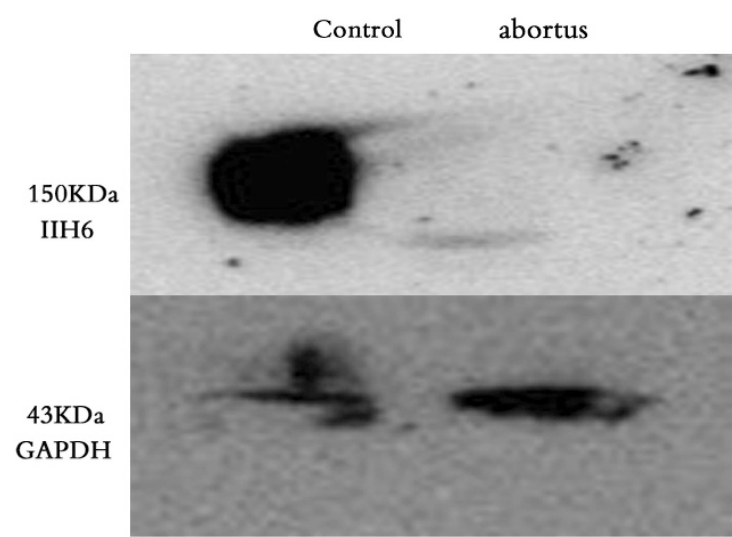

Figure 5 Western blot analysis of abortus muscle. Glycosylated $\alpha$-dystroglycan ( $\alpha$-DG) (150 kDa) was almost absent compared with the normal control. GAPDH, glyceraldehyde 3-phosphate dehydrogenase.

The POMT assay demonstrated that the severity of the clinical phenotype correlated with POMT activity.

Loss of POMT activity leads to $\alpha$-DG O-linked mannosylation defects. ${ }^{11,12}$ The generation of appropriate dystroglycanopathy mouse models has been hampered by early embryonic lethality in the Pomt1-null mouse, demonstrating the importance of protein O-mannosylation in development. ${ }^{13}$

Patient 1 presented with typical clinical signs of WWS and had two heterozygous mutations: c.313C > T/p.Arg105Cys and c.2208delG/p. Trp736*. The Arg105Cys mutation is located in the highly conserved protein mannosyltransferase catalytic domain, and has previously been associated with a WWS clinical phenotype. ${ }^{5}$ The c.2208delG/p. Trp736* mutation has been reported in mild muscular dystrophy; ${ }^{6}$ therefore, c.313C $>$ T/p.Arg105Cys might have a crucial role in the severe phenotype.

Patients 2 and 3 both had the heterozygous c.1457G $>\mathrm{C} / \mathrm{p}$. Trp486Ser mutation, which is located in the MIR domain. The second mutation in Patient 2 was a splice site mutation, c.1052+1G $>$ A, whereas that in Patient 3 was c.130G $>$ A/p.Glu44Lys. The clinical phenotypes of Patients 2 and 3 were mild CMD with mental retardation. They could run for a few minutes but were easily fatigued, and could attend school but found studying difficult. There were also some differences between the two: Patient 2 had obviously abnormal brain structure, including 'cobblestone' lissencephaly, whereas Patient 3 had much milder structural abnormalities of the brain.

The clinical phenotypes of Patients 4 and 5 conformed to severe CMD with mental retardation. Patient 4 had a one amino-acid deletion mutation in exon 3, c.180-182del/p.Pro60del, and a second mutation of c.1958C > A/p.Pro653Gln. The c.180 - 182del/p.Pro60del mutation has been reported previously, but it is not known whether it is a pathogenic mutation. ${ }^{14}$ c.1958C $>\mathrm{A} / \mathrm{p}$.Pro653Gln has also been reported previously and is associated with the CMD phenotype. ${ }^{15}$ Patient 4 had obvious global developmental delay as well as mental retardation, but his brain MRI was normal, which is consistent with the findings for the previously reported patient with the c.1958C > A/p.Pro653Gln mutation. ${ }^{15}$ Two of the mutations in Patient 5 have not been previously reported. c. $-32 \mathrm{~A}>\mathrm{G}$ is a splice site mutation in the POMT1 5'-untranslated region and the other, a frame shift mutation, c.2207delG, involves the same amino-acid change as a mutation in Patient 1. Patient 5 was unable to walk unsupported until he was 8 years old. To investigate whether c. $-32 \mathrm{~A}>\mathrm{G}$ is a pathogenic mutation or not, we examined 200 normal control chromosomes but did not identify the mutation. Furthermore, the c. $-32 \mathrm{~A}>\mathrm{G}$ mutation is not present in the 1000 Genomes Database (http://www.1000genomes.org). The reduced POMT activity and decreased $\alpha$-DG glycosylation seen in Patient 5 further indicate the pathogenicity of these mutations.

We also performed prenatal diagnosis on two pregnancies of the mother of Patient 5 (subsequent to that of Patient 5). In the first pregnancy, the fetus had the same mutation as Patient 5 , leading to its abortion. Western blotting confirmed that glycosylated $\alpha-D G$ was absent in the muscle of the abortus.

Dermal fibroblasts may be used to facilitate the diagnosis of dystroglycanopathy as well as to study the pathogenic mechanism of POMT1 mutations. The severity of the clinical phenotype has been shown to be inversely correlated with POMT activity, ${ }^{16}$ and our study confirmed this point. We examined POMT activity in dermal fibroblasts from Patients 2-5, and found that POMT activity in patients with mild CMD with mental retardation (Patients 2 and 3) was lower compared with that in the normal control but higher compared with that in patients with severe CMD with mental retardation (Patients 4 and 5). To our knowledge, there have been no previous reports on muscle POMT activity in WWS or CMD patients; however, we measured muscle POMT activity of an abortus and found it to be absent compared with the normal control.

Based on our results, we conclude that different mutation combinations lead to wholly different clinical phenotypes. There is also cardiac involvement in patients carrying POMT1 mutations; ${ }^{17}$ however, only Patient 3 presented a cardiac phenotype (left ventricular mild enlargement), which further supports the notion of variable phenotypes with respect to different POMT1 mutations. For the other patients without cardiac abnormalities, continuous follow-up is necessary. 
POMT1 is mainly expressed in regions that are critical for proper brain and cerebellar development. ${ }^{8}$ The genotype-phenotype correlation could be related to the position of the mutation within the gene; the protein mannosyltransferase catalytic domain mutation produced a more severe phenotype than mutations in other positions. ${ }^{18}$ There were also reports showing that mutations within the protein mannosyltransferase and protein mannosyltransferase 4TMC domain tended to be associated with more severe presentation. ${ }^{19}$

Previously, it was believed that POMT1 mutations were the most common cause of WWS, but several other genes have recently been shown to be involved in WWS, such as isoprenoid synthase domain-containing, glycosyltransferase-like domain-containing 2 and TM protein $5,{ }^{20-24}$ and POMT1 mutations also led to varying disease phenotypes. In this report, all of the patients had different mutations, and their clinical phenotypes differed.

Our study emphasizes the importance of genetic testing in patients with CMD to confirm gene involvement, and thus inform family counseling and prenatal diagnostic testing. Furthermore, assaying POMT activity in dermal fibroblasts may be a helpful way to more accurately evaluate the prognosis in young patients with POMT1 mutations.

\section{CONFLICT OF INTEREST}

The authors declare no conflict of interest.

\section{ACKNOWLEDGEMENTS}

We are grateful to all the patients and their families who have participated in this study. This work was supported by grants from the National Natural Science Foundation of China (81271400), the National Basic Research Program of China (2012CB944602), Grants-in-Aid for Scientific Research on Innovative Areas (26110727), Grants-in-Aid for Scientific Research (B) (25293016) and a Grant-in-Aid for Intramural Research (26-8) for Neurological and Psychiatric Disorders of NCNP.

1 Jurado, L. A., Coloma, A. \& Cruces, J. Identification of a human homolog of the Drosophila rotated abdomen gene (POMT1) encoding a putative protein 0-mannosyl-transferase, and assignment to human chromosome 9q34.1. Genomics 58 171-180 (1999).

2 Willer, T., Amselgruber, W., Deutzmann, R. \& Strahl, S. Characterization of POMT2, novel member of the PMT protein 0-mannosyltransferase family specifically localized to the acrosome of mammalian spermatids. Glycobiology 12, 771-783 (2002).

3 Hewitt, J. E. Abnormal glycosylation of dystroglycan in human genetic disease Biochim. Biophys. Acta 1792, 853-861 (2009).

4 Beltrán-Valero de Bernabé, D., Currier, S., Steinbrecher, A., Celli, J., van Beusekom, E., van der Zwaag, B. et al. Mutations in the O-mannosyltransferase gene POMT1 give rise to the severe neuronal migration disorder Walker-Warburg syndrome. Am. J. Hum Genet. 71, 1033-1043 (2002).

5 van Reeuwijk, J., Maugenre, S., van den Elzen, C., Verrips, A., Bertini, E., Muntoni, F. et al. The expanding phenotype of POMT1 mutations: from Walker-Warburg syndrome to congenital musculardystrophy, microcephaly, and mental retardation. Hum. Mutat. 27, 453-459 (2006).

6 Al-Zaidy, S. A., Baskin, B., Hawkins, C., Yoon, G., Ray, P. N. \& Vajsar, J. Milder phenotype of congenital muscular dystrophy in a novel POMT1 mutation. Muscle Nerve 45, 752-755 (2012).

7 Balci, B., Uyanik, G., Dincer, P., Gross, C., Willer, T., Talim, B. et al. An autosomal recessive limb girdle muscular dystrophy (LGMD2) with mild mental retardation is allelic to Walker-Warburg syndrome (WWS) caused by a mutation in the POMT1 gene. Neuromuscul. Disord. 15, 271-275 (2005).

8 Vajsar, J. \& Schachter, H. Walker-Warburg syndrome. Orphanet. J. Rare Dis. 1, 29 (2006).

9 Hafner, P., Bonati, U., Fischmann, A., Schneider, J., Frank, S., Morris-Rosendahl, D. J. et al. Skeletal muscle MRI of the lower limbs in congenital muscular dystrophy patients with novel POMT1 and POMT2 mutations. Neuromuscul. Disord. 24, 321-324 (2014).

10 Manya, H., Chiba, A., Yoshida, A., Wang, X., Chiba, Y., Jigami, Y. et al. Demonstration of mammalian protein O-mannosyltransferase activity: coexpression of POMT1 and POMT2 required for enzymatic activity. Proc. Natl Acad. Sci. USA 101 500-505 (2004).

11 Akasaka-Manya, K., Manya, H. \& Endo, T. Mutations of the POMT1 gene found in patients with Walker-Warburg syndrome lead to a defect of protein 0-mannosylation. Biochem. Biophys. Res. Commun. 325, 75-79 (2004).

12 Manya, H., Bouchet, C., Yanagisawa, A., Vuillaumier-Barrot, S., Quijano-Roy, S., Suzuki, Y. et al. Protein O-mannosyltransferase activities in lymphoblasts from patients with alpha-dystroglycanopathies. Neuromuscul. Disord. 18, 45-51 (2008).

13 Willer, T., Prados, B., Falcon-Perez, J. M., Renner-Müller, I., Przemeck, G. K., Lommel, M. et al. Targeted disruption of the Walker-Warburg syndrome gene Pomt1 in mouse results in embryonic lethality. Proc. Natl Acad. Sci. USA 101 14126-14131 (2004).

14 Bouchet, C., Gonzales, M., Vuillaumier-Barrot, S., Devisme, L., Lebizec, C., Alanio, E. et al. Molecular heterogeneity in fetal forms of type II lissencephaly. Hum. Mutat. 28 1020-1027 (2007).

15 Wallace, S. E., Conta, J. H., Winder, T. L., Willer, T., Eskuri, J. M., Haas, R. et al. A novel missense mutation in POMT1 modulates the severe congenital muscular dystrophy phenotype associated with POMT1 nonsense mutations. Neuromuscul. Disord. 24, 312-320 (2014)

16 Lommel, M., Cirak, S., Willer, T., Hermann, R., Uyanik, G., van Bokhoven, H. et al. Correlation of enzyme activity and clinical phenotype in POMT1-associated dystroglycanopathies. Neurology 74, 157-164 (2010).

17 Finsterer, J., Ramaciotti, C., Wang, C. H., Wahbi, K., Rosenthal, D., Duboc, D. et al. Cardiac findings in congenital muscular dystrophies. Pediatrics 126, 538-545 (2010).

18 Balci, B., Uyanik, G., Dincer, P., Gross, C., Willer, T., Talim, B. et al. An autosomal recessive limb girdle muscular dystrophy (LGMD2) with mild mental retardation is allelic to Walker-Warburg syndrome (WWS) caused by a mutation in the POMT1 gene. Neuromuscul. Disord. 15, 271-275 (2005).

19 Godfrey, C., Clement, E., Mein, R., Brockington, M., Smith, J., Talim, B. et al. Refining genotype phenotype correlations in muscular dystrophies with defective glycosylation of dystroglycan. Brain 130, 2725-2735 (2007)

20 Yoshida-Moriguchi, T., Willer, T., Anderson, M. E., Venzke, D., Whyte, T., Muntoni, F. et al. SGK196 is a glycosylation-specific O-mannose kinase required for dystroglycan function. Science 341, 896-899 (2013).

21 Willer, T., Lee, H., Lommel, M., Yoshida-Moriguchi, T., de Bernabe, D. B., Venzke, D. et al. ISPD loss-of-function mutations disrupt dystroglycan 0-mannosylation and cause Walker-Warburg syndrome. Nat. Gene 44, 575-580 (2012).

22 Roscioli, T., Kamsteeg, E. J., Buysse, K., Maystadt, I., van Reeuwijk, J., van den Elzen, C. et al. Mutations in ISPD cause Walker-Warburg syndrome and defective glycosylation of $\alpha$-dystroglycan. Nat. Genet. 44, 581-585 (2012).

23 Buysse, K., Riemersma, M., Powell, G., van Reeuwijk, J., Chitayat, D., Roscioli, T. et al. Missense mutations in $\beta-1,3-N$-acetylglucosaminyltransferase 1 (B3GNT1) cause Walker-Warburg syndrome. Hum. Mol. Genet. 22, 1746-1754 (2013).

24 Vuillaumier-Barrot, S., Bouchet-Séraphin, C., Chelbi, M., Devisme, L., Quentin, S. Gazal, S. et al. Identification of mutations in TMEM5 and ISPD as a cause of severe cobblestone lissencephaly. Am. J. Hum. Gene 91, 1135-1143 (2012) 